\title{
Microsurgical Management of Trigeminal Schwannoma: Cohort Analysis and Systematic Review
}

\author{
Maick Willen Fernandes Neves ${ }^{1}$ Paulo Henrique Pires de Aguiar ${ }^{2}$ Telmo Augusto Barba Belsuzarri ${ }^{1}$ \\ André Monteiro Soares de Araujo ${ }^{3}$ Samantha Lorena Paganelli ${ }^{4}$ Marcos Vinicius Calfat Maldaun ${ }^{5}$ \\ ${ }^{1}$ Department of Neurosurgery, Hospital Celso Pierro, Pontifícial \\ Catholic University of Campinas, Campinas, São Paulo, Brazil \\ 2 Department of Neurosurgery, Pontifícial Catholic University of \\ Sorocaba, Sorocaba, São Paulo, Brazil \\ ${ }^{3}$ Department of Neurosurgery, Pontifícial Catholic University of \\ Campinas, Campinas, São Paulo, Brazil \\ ${ }^{4}$ Department of Neurosurgery, Hospital Ramos Mejia, Buenos Aires \\ University, CABA, Argentina \\ ${ }^{5}$ Department of Neurosurgery, Santa Paula Hospital, São Paulo, \\ São Paulo, Brazil \\ Address for correspondence Maick Willen Fernandes Neves, MD, \\ Department of Neurosurgery, Pontifícial Catholic University of \\ Campinas, Campinas, SP 13083-970, Brazil \\ (e-mail: maickfernandes@gmail.com).
}

J Neurol Surg B 2019;80:264-269.

\begin{abstract}
Keywords

- brain tumor

- trigeminal schwannoma

- microsurgery

- radiosurgery

Background Trigeminal schwannomas are benign tumors with a predilection for women between 40 and 60 years of age and account for less than $0.5 \%$ of all intracranial tumors. Clinical presentation depends on size and location, and typical symptoms are ipsilateral hypesthesia, headache, and facial pain. Clinical features and imaging usually make the Diagnosis.

Methods A retrospective cohort analysis of 14 patients treated at our institution between January 1999 and October 2016 was performed, with a critical and systematic review of data from the literature, focusing on articles published over the same period. Results Fourteen patients were included in our series comprised of mostly women with a mean age of 40 years. Lesion size ranged from 3 to $6.5 \mathrm{~cm}$ (mean $4.6 \mathrm{~cm}$ ). Clinical status of patients was evaluated and also rated based on the Karnofsky Perfomance Scale with values greater than $90 \%$ found in all patients pre and postoperatively. Surgery was our treatment of choice, and gross total resection was achieved in $71 \%$ of patients. Associated morbidity was high at $57 \%$, predominantly from cranial nerve palsy, and no deaths were encountered.

Conclusions Microsurgery is a suitable treatment for large symptomatic trigeminal schwannomas, achieving good local control rates over the observation period at acceptable morbidity. Therefore, microsurgery appears to a suitable option. During the study period, a significant evolution in the availability of treatment methods occurred. Radiosurgery emerged as an alternative treatment for unresectable lesions. A comprehensive review of the available literature was performed, and results were compared according to treatment modality.
\end{abstract}

received

April 25, 2018

accepted after revision

July 11, 2018

published online

August 28, 2018 (c) 2019 Georg Thieme Verlag KG

Stuttgart · New York
DOI https://doi.org/ 10.1055/s-0038-1669418. ISSN 2193-6331. 


\section{Introduction}

Trigeminal schwannomas (TS) are benign neoplasms originating from the nerve sheath, distal to the schwann-oligodendroglia junction. ${ }^{1}$ These tumors of the skull base account for 0.07 to $0.36 \%$ of intracranial tumors and 1 to $8 \%$ of adult intracranial schwannomas, but they can make up around $10 \%$ of schwannomas encountered in adolescence. ${ }^{2-6}$ This correlates to an approximate incidence of 1 case per 100,000 inhabitants, affecting predominantly women between the fourth and sixth decade of life. Malignant TS are very infrequently reported and mainly occur associated with neurofibromatosis type $1 .^{7}$

The clinical presentation of these tumors depends on location and size; however, it is common to see trigeminal hypesthesia, facial pain, and headaches, sometimes in combination with hearing impairment, seizures, diplopia, ataxia, hemiparesis, papilledema, jaw weakness, obstructive hydrocephalus, facial paralysis, and hemifacial spasm. ${ }^{6,8-12}$

Diagnosis is obtained from the clinical history, with magnetic resonance imaging (MRI) as the imaging modality of choice. Characteristic lesional radiographic features are a hypointense signal on $\mathrm{T} 1$, a hyperintense signal on $\mathrm{T} 2$, and avid contrast uptake after intravenous gadolinium injection. Computed tomography (CT) scanning of the skull is a complimentary exam aiding in the assessment of the skull base anatomy for surgical planning. ${ }^{2}$

\section{Material and Method}

A retrospective analysis was performed of a cohort consisting of 14 patients with TS treated at the Department of Neurosurgery of Santa Paula Hospital in São Paulo, Brazil from January 1999 to October 2016. Our data were then compared with results from published articles, after performing a systematic review and meta-analysis. A PubMed search was done from October to December 2016, using the keywords "Brain Tumor", "Trigeminal Schwannoma”, "Microsurgery", and "Radiosurgery." We extracted relevant data and created tables for a pooled analysis for comparison.

\section{Classification}

Lesions were classified according to 5 anatomically discernible segments of the nerve: (1) intra-axial (brainstem), (2) cisternal, (3) Meckel's cave, (4) cavernous sinus, and (5) skull base/extracranial. Tumors were further characterized according to their composition into solid, cystic, or combined solid-cystic lesions. ${ }^{2}$ They were subdivided according to size into small $(<3 \mathrm{~cm})$, medium $(3-4 \mathrm{~cm})$, large $(4-5 \mathrm{~cm})$, and giant $(>5 \mathrm{~cm}) .{ }^{6}$ Another classification system was introduced by Samii and colleagues, ${ }^{12}$ who classified tumors based on radiographic criteria into 4 types: Type $\mathrm{A}=$ intracranial tumors, predominantly in the middle fossa; Type $\mathrm{B}=$ intracranial tumors, predominantly in the posterior fossa; Type $\mathrm{C}=$ intracranial dumbbell-shaped tumors in the middle and posterior fossa; Type $\mathrm{D}=$ extracranial tumors with intracranial extensions (-Figs. 1-4).

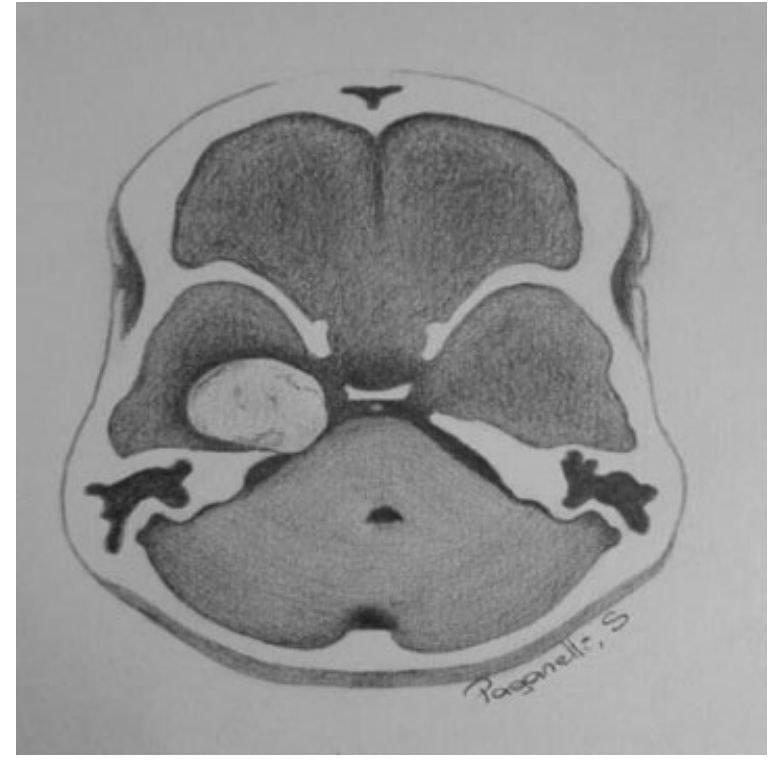

Fig. 1 Type A, intracranial tumor predominantly in the middle fossa.

\section{Results}

During the study period, 14 adult patients were treated microsurgically. The cohort consisted of 13 female and 1 male patients. The mean age of the cohort was 40 years (range: 19-65 years). Based on size, 5 of the lesions were classified as medium in size, 6 as large and 3 as a giant. Lesions were located on the left side in $9 / 14$ patients (64\%) All patients were in good clinical status (Karnofsky Perfomance Scale [KPS] > 90) with mild functional compromise only, but showed a high incidence of headaches and vomiting (-Table $\mathbf{1}$ ). The most frequent clinical symptom was headaches, followed by hypesthesia in 1 of the trigeminal branches (see - Table 2 ).

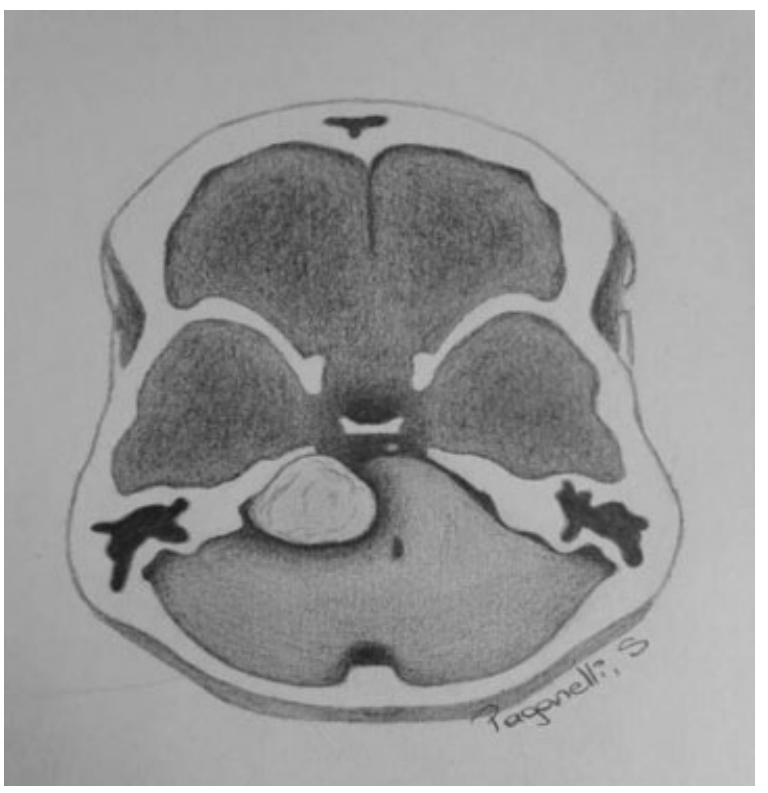

Fig. 2 Type B, intracranial tumor predominantly in the posterior fossa. 


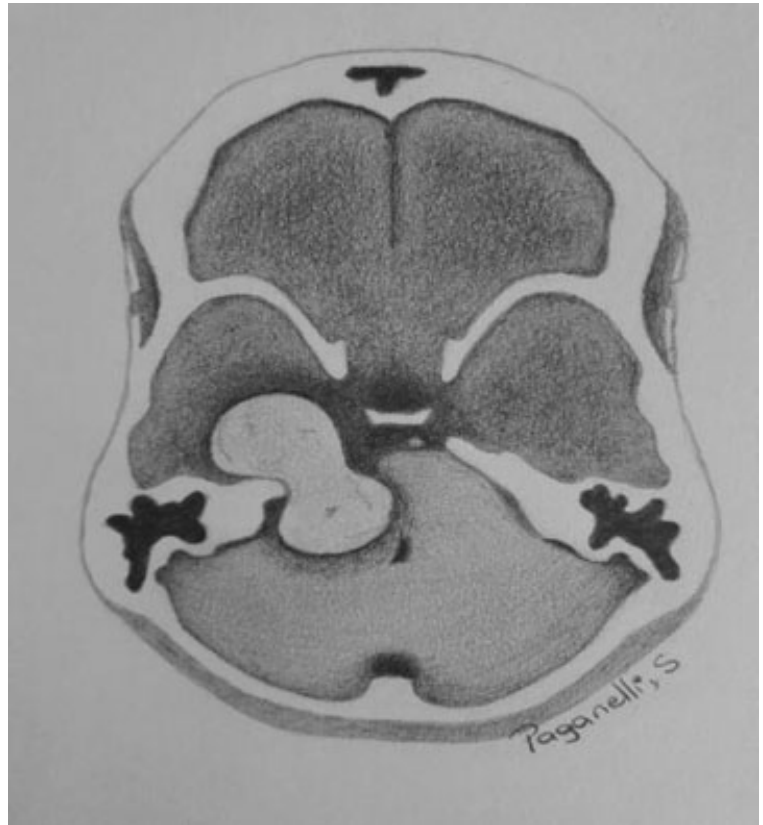

Fig. 3 Type C, intracranial dumbbell-shaped tumor in the middle and posterior fossa.

Tumors were predominantly cystic in $12 / 14$ patients (85\%) and had an average size of $4.6 \mathrm{~cm}$ (see - Fig. 5). Three out of 14 patients (21\%) presented with symptomatic hydrocephalus, requiring the placement of a ventriculoperitoneal shunt. One of those patients, showed worsening signs of intracranial hypertension during the follow-up period, while the other 2 had a resolution of their hydrocephalic symptoms after the shunting procedure.

During the postoperative period, 1 patient developed wound infection, and 8 patients showed some form of cranial nerve palsy and 2 patients had surgical wound hemorrhage.

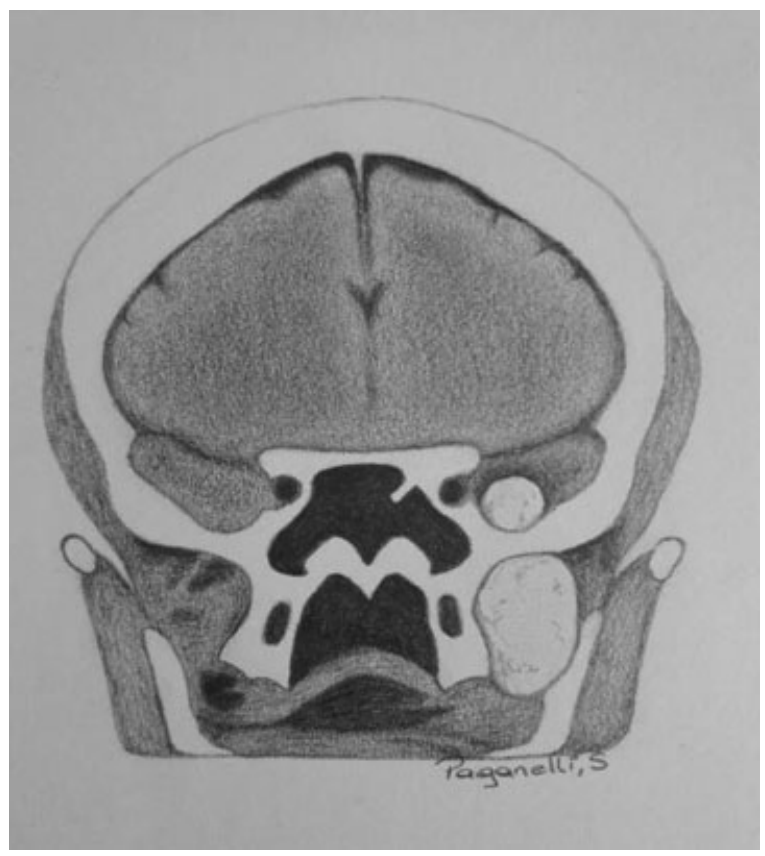

Fig. 4 Type D, extracranial tumor with intracranial extensions.
None of these cases required a second operation, but was manageable expectantly.

Based on presurgical imaging, 3 patients of our cohort presented with type A lesions (predominantly located in the middle fossa; $21 \%$ and 11 tumors were intracranial dumbbellshaped type $C$ tumors, mainly located in the middle and posterior fossa (78\%). Gross total resection was achieved in 10 patients ( $71 \%$ of cases) based on intraoperative assessment and postoperative MRI (see - Fig. 6). Patients were regularly followed up after surgery with MRI and clinical visits, with recurrences observed in 3 cases ( 2 after subtotal resection and 1 after an alleged gross total resection). Further surgery was necessary for 1 of these 3 patients. All the 3 patients with residual disease subsequently underwent stereotaxic radiosurgery, gamma-knife, for adjuvant treatment.

\section{Discussion}

The introduction and improvement of available microscopic techniques and neuroanesthesia have decreased the morbidity and mortality of patients undergoing surgery for trigeminal schwannomas. ${ }^{13}$ Over the years, complete resection with minimal morbidity and mortality has become the goal of treatment with an emphasis on maintaining the quality of life in TS patients.

Complete resection is the most aggressive form of surgical treatment and offers potentially a cure or prolonged progression-free survival, but is frequently associated with a high incidence of neurological deficits.

\section{Surgical}

TS microsurgery aims at achieving a complete resection of the lesion and should be performed without adding any new neurological deficit. However, incomplete resections have been noted to be mostly related to inadequate exposure or complex tumor invasion of the cavernous sinus or dense tumor adherence to the brainstem. ${ }^{9,14-16}$ Due to improved microsurgical techniques, tumor recurrence rates are gradually decreasing and range from 0 to $17 \%{ }^{17}$ For tumors smaller than $3 \mathrm{~cm}$, gross total resection rates of more than $95 \%$ have been reported. ${ }^{11}$ However, if tumor recurrence occurs, it usually does so in the cavernous sinus and Meckel's cave. ${ }^{17}$

Surgical approaches were based on the classification of the lesion and are described in $\boldsymbol{-}$ Table $\mathbf{1}$, while comparable results from the literature are shown in -Table 3. Thus, for type A tumors, surgeons may use a pterional, orbitozygomatic, or subtemporal approach. Type B tumors can be best approached via a retrosigmoid craniotomy (in particular, if they are almost entirely in the posterior fossa). If a significant portion of the tumor is located in the middle fossa, a transpetrous approach, or a combination of approaches must be used. Type $C$ tumors are the most frequent tumor type reported. These TS are complex and usually require combined approaches to the middle fossa, most commonly an anterior petrosectomy, and posterior fossa, as posterior petrosectomy, and retrosigmoid approach. ${ }^{18}$ Type D tumors can be accessed using a preauricular infratemporal approach. ${ }^{5}$ Lesions that reach peripherally into the maxillary sinus and originate from the middle fossa or 
Table 1 Case series

\begin{tabular}{|l|l|l|l|l|l|l|l|}
\hline Case/gender & Age $(\mathbf{y})$ & KPS $(\%)$ & Grade of resection & Follow-up & Side & Size $(\mathbf{c m})$ & Classification \\
\hline $1 / \mathrm{M}$ & 35 & 100 & GTR & $17 \mathrm{y}$ & $\mathrm{L}$ & 4.5 & $\mathrm{C}$ \\
\hline $2 / \mathrm{F}$ & 54 & 90 & GTR & $16 \mathrm{y}$ & $\mathrm{L}$ & 5 & $\mathrm{C}$ \\
\hline $3 / \mathrm{F}$ & 65 & 100 & GTR & $10 \mathrm{y}$ & $\mathrm{R}$ & 4.5 & $\mathrm{C}$ \\
\hline $4 / \mathrm{F}$ & 52 & 100 & GTR & $8 \mathrm{y}$ & $\mathrm{R}$ & 5 & $\mathrm{C}$ \\
\hline $5 / \mathrm{F}$ & 19 & 100 & SR & $8.5 \mathrm{y}$ & $\mathrm{L}$ & 4 & $\mathrm{C}$ \\
\hline $6 / \mathrm{F}$ & 34 & 100 & GTR & $7 \mathrm{y}$ & $\mathrm{R}$ & 4 & $\mathrm{~A}$ \\
\hline $7 / \mathrm{F}$ & 43 & 100 & GTR & $6.5 \mathrm{y}$ & $\mathrm{L}$ & 6 & $\mathrm{C}$ \\
\hline $8 / \mathrm{F}$ & 32 & 100 & SR & $6 \mathrm{y}$ & $\mathrm{L}$ & 4.5 & $\mathrm{~A}$ \\
\hline 9/F & 22 & 90 & SR & $6 \mathrm{y}$ & $\mathrm{R}$ & 6.5 & $\mathrm{C}$ \\
\hline $10 / \mathrm{F}$ & 28 & 100 & SR & $6 \mathrm{y}$ & $\mathrm{L}$ & 4 & $\mathrm{C}$ \\
\hline $11 / \mathrm{F}$ & 19 & 100 & GTR & $6 \mathrm{y}$ & $\mathrm{R}$ & 3 & $\mathrm{C}$ \\
\hline $12 / \mathrm{F}$ & 53 & 100 & GTR & $2 \mathrm{y}$ & $\mathrm{L}$ & 4.5 & $\mathrm{C}$ \\
\hline $13 / \mathrm{F}$ & 55 & 100 & GTR & $1 \mathrm{y}$ & $\mathrm{L}$ & 4 & $\mathrm{~A}$ \\
\hline $14 / \mathrm{F}$ & 65 & 100 & GTR & $2 \mathrm{mo}$ & $\mathrm{L}$ & 6 & $\mathrm{C}$ \\
\hline
\end{tabular}

Abbreviations: GTR, gross total resection; L, left; mo, month; R, right; SR, subtotal resection; y, year.

Table 2 Signs and symptoms presented in the preoperative

\begin{tabular}{|l|l|}
\hline Headache & $50 \%$ \\
\hline Vomit & $21 \%$ \\
\hline Behavior & $14 \%$ \\
\hline Strabismus & $14 \%$ \\
\hline Tinnitus & $14 \%$ \\
\hline Papilledema & $21 \%$ \\
\hline Hypoesthesia & $28 \%$ \\
\hline Paresis III & $7 \%$ \\
\hline Paresis V & $14 \%$ \\
\hline Paresis VII & $14 \%$ \\
\hline Pyramidal signs & $7 \%$ \\
\hline
\end{tabular}

cavernous sinus, can be accessed by a combination of transmaxillary and middle fossa approaches. ${ }^{6}$

We believe that the best results can be achieved with small-sized lesions and tumor configurations of types A or B. However, our series did not present with any lesion classified as small $(<3 \mathrm{~cm})$, and only 3 type-A cases were encountered.

According to the literature, complications depend on size, location and approaches chosen, but the most frequently reported postoperative complications were: meningitis, fluid fistulas, masseter muscle atrophy, trigeminal pain and facial paresthesia, in addition to paresis of the cranial nerves III-VI. ${ }^{17}$

Due to high surgical morbidity seen with TS resections, the field is progressively evolving toward minimally invasive techniques.

Table 3 Trigeminal schwannoma case series comparison

\begin{tabular}{|l|l|l|l|l|l|l|l|l|l|}
\hline Author & Y & $\begin{array}{l}\text { Radical } \\
\text { removal (\%) }\end{array}$ & $\begin{array}{l}\text { Mortality } \\
(\%)\end{array}$ & $\begin{array}{l}\text { Morbility } \\
(\%)\end{array}$ & Type A & Type B & Type C & Type D & Total \\
\hline Yoshida and Kawase $^{18}$ & 1999 & $20(74 \%)$ & 0 & 74 & 4 & 5 & 10 & 8 & 27 \\
\hline Goel et al $^{10}$ & 2003 & $51(70 \%)$ & 3 & 7 & 29 & 7 & 30 & 7 & 73 \\
\hline Bhawani et al $^{3}$ & 2006 & $35(76 \%)$ & 1 & 15 & 0 & 0 & 57 & 0 & 57 \\
\hline Pamir et al $^{23}$ & 2007 & $17(94 \%)$ & 0 & 28 & 5 & 2 & 9 & 2 & 18 \\
\hline Fukaya et al $^{7}$ & 2010 & $46(81 \%)$ & 2 & 68 & 15 & 12 & 26 & 4 & 57 \\
\hline Wanibuchi et al & 2012 & $86(82 \%)$ & 0 & 9 & 39 & 22 & 33 & 14 & 105 \\
\hline Chen et al $^{9}$ & 2014 & $52(95 \%)$ & 0 & 5 & 13 & 10 & 21 & 11 & 55 \\
\hline Samii et al & 2014 & $15(75 \%)$ & 0 & 4 & 8 & 1 & 8 & 4 & 20 \\
\hline Jeong et al & & 2014 & $47(95.9 \%)$ & 0 & 18 & 20 & 20 & 9 & 0 \\
\hline Current study & 2016 & $10(71 \%)$ & 0 & 3 & 3 & 0 & 11 & 0 \\
\hline
\end{tabular}




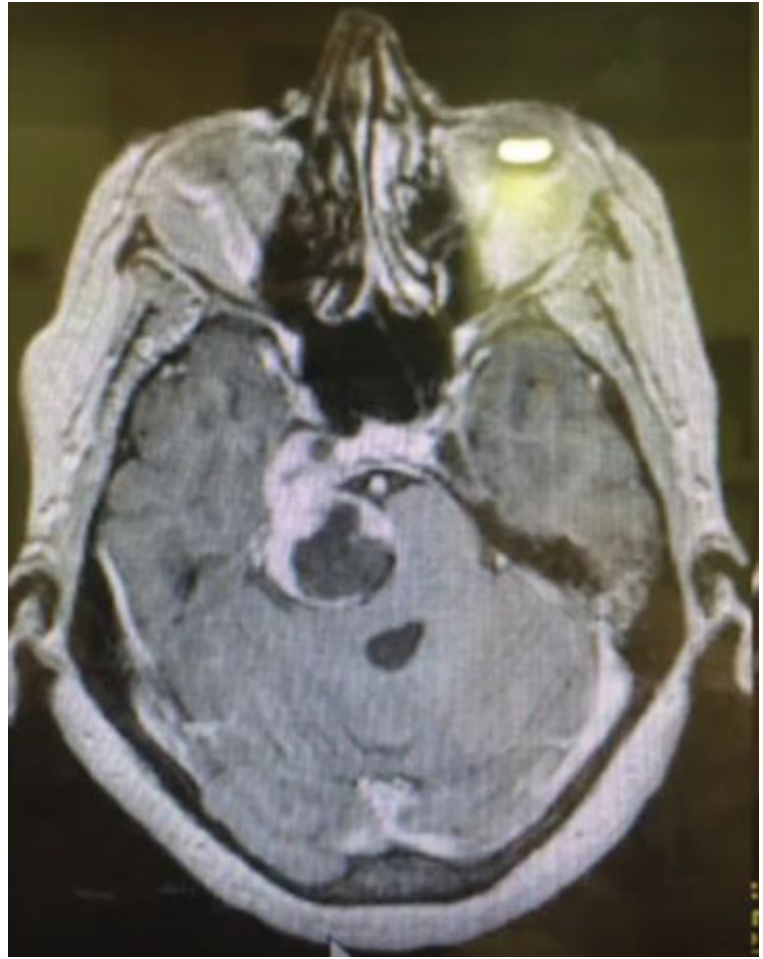

Fig. 5 Magnetic resonance imaging (MRI) in axial sections with contrast, preoperative.

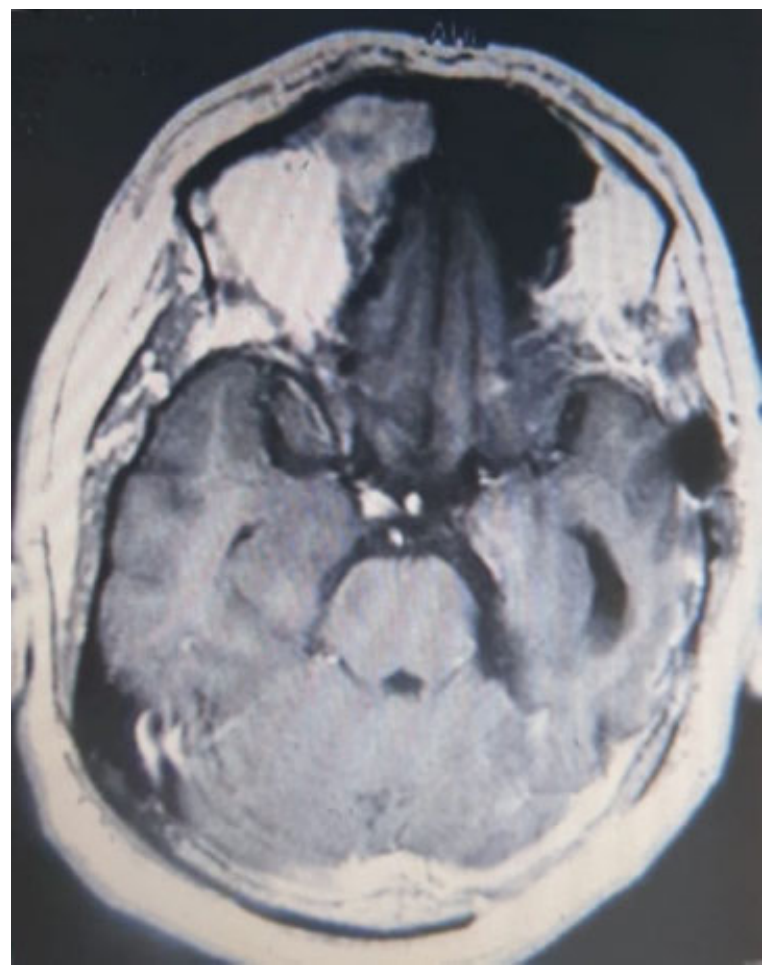

Fig. 6 MRI, T1 with contrast, demonstrating gross subtotal resection (GSR).

\section{Biopsy}

Percutaneous biopsy is rarely employed, but offers a diagnostic alternative for lesions in Meckel's Cave or lesions along the third division of the trigeminal nerve. The biopsy can be performed employing a percutaneous technique comparable to approaches used for trigeminal neuralgia ${ }^{18-20}$ and can be done before any adjuvant treatment (e.g., SRS). We want to emphasize the relevance of this approach as published by Sindou and colleagues, who reported on a cohort of 50 patients, who underwent a percutaneous biopsy. Among these 50 cases, which were biopsied due to insufficient imaging features to set the correct diagnosis, the authors identified only the case 3 schwannomas exemplifying the importance of this minor procedure for avoiding unnecessary open surgery, when other nonsurgical pathologies are found. ${ }^{20,21}$

\section{Endoscopic Endonasal}

More recently, endoscopic transnasal surgical (ETS) approaches have been reported as suitable for lesions affecting branches of V2 and V3. This technique allows accessing tumors that are located paramedian, in the parasellar region, in Meckel's cave, as well as lesions with discrete extension into the posterior fossa. ${ }^{1}$ This technique can also be used for lesions in the middle fossa, pterygopalatine fossa, and infratemporal fossa. ${ }^{1,19}$ Arguments in favor of this approach are less retraction on temporal structures or the cerebellum and good access to structures in the vicinity of the brainstem. However, risk factors associated with ETS include a higher rate of CSF (cerebrospinal fluid) liquorrhea and a potential risk of vascular complications due to the topographic relation of the tumor to the internal carotid artery. ${ }^{6}$

\section{Radiosurgery}

Radiosurgery aims to control tumor growth without open surgical access and hence intends to minimize any new treatment associated neurological deficits. To achieve this goal, focal irradiation is delivered on the lesion, ${ }^{22,23}$ with the aim to arrest further growth and possibly induce tumor necrosis and shrinking of the mass. Radiosensitivity of the tumor depends on a variety of factors, including its composition. Less fibrous tumors with higher cellularity and with cystic areas are better candidates for gamma-knife surgery. ${ }^{11}$ Some studies have shown an average decrease of TS size by approximately $6.8 \mathrm{~mL}$, over a follow-up of a period of 44 months. ${ }^{4,22}$ In our series, 3 young patients, who received a partial resection due to complications risk, were treated with gamma-knife surgery, once they presented MIB- 1 above $3 \%$, and so they had a higher risk of regrowth and possibly the need of a new posterior surgery. ${ }^{2,24}$

The advancement of modern neuro-oncology increasingly relies on molecular studies, obtained from the surgical specimen. When noninvasive SRS treatment is chosen empirically, the indication is based on a clinical and radiological diagnosis alone, which carries the risk of a misdiagnosis, since the differential diagnoses for tumors in this location that includes other entities, such as meningiomas, epidermoid cysts, metastasis, chondrosarcomas, chordomas, chondromas, other schwannomas, and maxillary sinus tumors. One needs to be aware of the fact that such treatment without available histopathology may thus occasionally lead to inadequate initial treatment or delay in obtaining the correct diagnosis. ${ }^{21,25}$ 
Albeit rare, another problem is posed by malignant trigeminal schwannomas, which may present with the local disease, as well as systemic hematogenous dissemination to the lung and bone in up to one-third of patients. ${ }^{7}$ SRS is a local treatment and therefore, unsuitable to address this potentially life-threatening diagnosis. One has to remain vigilant about this diagnosis and potential metastases during follow-up.

\section{Conclusion}

In this study, we report our experience of microsurgical treatment in a sizeable cohort of patients with trigeminal schwannomas and conclude that the best treatment for large symptomatic lesions remains open surgery. It allows for a gross total resection and provides the patient with immediate relief of symptoms of mass effect. It offers the patient a surgical cure and if exposure is optimal can often be performed without adding significant new neurological deficits. In cases of subtotal resection (STR), radiosurgery should be added to achieve a prolonged progression-free survival. Gentle STR in conjunction with SRS may also result in lower overall treatment morbidity and allow for a better quality of life posttreatment. Satisfactory results have also been achieved with primary radiosurgery for smaller lesions (less than $30 \mathrm{~mm}$ ), as well as for lesions that are unresectable.

Financial Support and Sponsorship

None.

Conflicts of Interest

There are no conflicts of interest.

\section{References}

1 Peker S, Bayrakli F, Kiliç T, Pamir MN. Gamma-knife radiosurgery in the treatment of trigeminal schwannomas. Acta Neurochir (Wien) 2007;149(11):1133-1137, discussion 1137

2 Aguiar PH, Tatagiba M, Samii M, Dankoweit-Timpe E, Ostertag H. The comparison between the growth fraction of bilateral vestibular schwannomas in neurofibromatosis 2 (NF2) and unilateral vestibular schwannomas using the monoclonal antibody MIB 1 . Acta Neurochir (Wien) 1995;134(1-2):40-45

3 Sharma BS, Ahmad FU, Chandra PS, Mahapatra AK. Trigeminal schwannomas: experience with 68 cases. J Clin Neurosci 2008;15 (07):738-743

4 Cantini R, Giorgetti W, Valleriani AM, Burchianti M. Trigeminal schwannomas in adolescence. Pediatr Neurosci 1987;13(04): 198-201

5 Sun J, Zhang J, Yu X, et al. Stereotactic radiosurgery for trigeminal schwannoma: a clinical retrospective study in 52 cases. Stereotact Funct Neurosurg 2013;91(04):236-242

6 Raza SM, Amine MA, Anand V, Schwartz TH. Endoscopic endonasal resection of trigeminal schwannomas. Neurosurg Clin N Am 2015;26(03):473-479
7 Jusué-Torres I, Martinez-Gutierrez JC, Elder BD, Olivi A. Giant trigeminal schwannoma presenting with obstructive hydrocephalus. Cureus 2015;7(11):e386

8 Niranjan A, Barnett S, Anand V, Agazzi S. Multimodality management of trigeminal schwannomas. J Neurol Surg B Skull Base 2016;77(04):371-378

9 Chen LF, Yang Y, Yu XG, et al. Operative management of trigeminal neuromas: an analysis of a surgical experience with 55 cases. Acta Neurochir (Wien) 2014;156(06):1105-1114

10 Goel A, Muzumdar D, Raman C. Trigeminal neuroma: analysis of surgical experience with 73 cases. Neurosurgery 2003;52(04): 783-790, discussion 790

11 Yianni J, inca EB, Rowe J, Radatz M, Kemeny AA. Stereotactic radiosurgery for trigeminal schwannomas. Acta Neurochir (Wien) 2012;154(02):277-283

12 Samii M, Migliori MM, Tatagiba M, Babu R. Surgical treatment of trigeminal schwannomas. J Neurosurg 1995;82(05):711-718

13 Stone JA, Cooper H, Castillo M, Mukherji SK. Malignant schwannoma of the trigeminal nerve. AJNR Am J Neuroradiol 2001;22(03): 505-507

14 Rangel-Castilla L, Russin JJ, Spetzler RF. Surgical management of skull base tumors. Rep Pract Oncol Radiother 2016;21(04): 325-335

15 Zhang L, Yang Y, Xu S, Wang J, Liu Y, Zhu S. Trigeminal schwannomas: a report of 42 cases and review of the relevant surgical approaches. Clin Neurol Neurosurg 2009;111(03):261-269

16 Wanibuchi M, Fukushima T, Zomordi AR, Nonaka Y, Friedman AH. Trigeminal schwannomas: skull base approaches and operative results in 105 patients. Neurosurgery 2012;70(1, Suppl operative):132-143, discussion 143-144

17 Samii M, Alimohamadi M, Gerganov V. Endoscope-assisted retrosigmoid intradural suprameatal approach for surgical treatment of trigeminal schwannomas. Neurosurgery 2014;10(Suppl4):565-575, discussion 575

18 Yoshida K, Kawase T. Trigeminal neurinomas extending into multiple fossa: surgical methods and review of the literature. J Neurosurg 1999;91(02):202-211

19 Agarwal A. Intracranial trigeminal schwannoma. Neuroradiol J 2015;28(01):36-41

20 Sindou M, Messerer M, Alvernia J, Saint-Pierre G. Percutaneous biopsy through the foramen ovale for parasellar lesions: surgical anatomy, method, and indications. Adv Tech Stand Neurosurg 2012;38(38):57-73

21 Jeong SK, Lee EJ, Hue YH, Cho YH, Kim JH, Kim CJ. A suggestion of modified classification of trigeminal schwannomas according to location, shape, and extension. Brain Tumor Res Treat 2014;2(02): 62-68

22 Champ CE, Mishra MV, Shi W, et al. Stereotactic radiotherapy for trigeminal schwannomas. Neurosurgery 2012;71(02):270-277, discussion 277

23 Pamir MN, Peker S, Bayrakli F, Kiliç T, Ozek MM. Surgical treatment of trigeminal schwannomas. Neurosurg Rev 2007;30(04): 329-337, discussion 337

24 Fukuda M, Oishi M, Hiraishi T, Natsumeda M, Fujii Y. Clinicopathological factors related to regrowth of vestibular schwannoma after incomplete resection. J Neurosurg 2011;114(05): 1224-1231

25 Ramina R, Mattei TA, Sória MG, et al. Surgical management of trigeminal schwannomas. Neurosurg Focus 2008;25(06):E6, discussion E6 